\title{
The effect of noncontingent outcomes on extinction of the response-outcome association
}

\author{
RUTH M. COLWILL \\ Brown University, Providence, Rhode Island
}

\begin{abstract}
The effect of noncontingent outcomes on an instrumental response-outcome $(\mathrm{R}-\mathrm{O})$ association was examined in four experiments using transfer tests. In each experiment, rats were first given instrumental discrimination training designed to establish different stimuli as signals $(\mathrm{S}+\mathrm{s})$ for different outcomes. Transfer responses were subjected to different treatments across the experiments and then tested with the S+s. In Experiments 1 and 2, two transfer responses were both initially trained with two contingent outcomes. Then, each transfer response was subjected either to the addition of noncontingent presentations of one of those outcomes (Experiment 1) or to the replacement of one of the contingent outcomes with noncontingent presentations of that outcome (Experiment 2). Transfer tests revealed no significant difference in the ability of an S+ to promote performance of a transfer response based on their shared association with either the contingent or the noncontingent outcome. These results suggest that a response reinforced with two outcomes remains equally well associated with both of those outcomes despite prolonged exposure to noncontingent presentations of one of those outcomes. In Experiments 3 and 4, the possibility that the noncontingent schedules of reinforcement used in Experiments 1 and 2 might be capable of establishing an association between a response and its noncontingent outcome was examined. Transfer responses were trained with one contingent outcome and a different noncontingent outcome. Performance of these transfer responses was augmented more by presentations of an $\mathrm{S}+$ trained with the contingent outcome than with the noncontingent outcome. These results confirm previous reports that instrumental responses are sensitive to outcome contingencies in acquisition and that noncontingent outcome presentations do not weaken previously established $\mathrm{R}-\mathrm{O}$ associations. Several explanations are considered for the failure of subsequent noncontingent presentations of an outcome to reduce the strength of its association with the instrumental response.
\end{abstract}

Two recent findings endorse the view that, over the course of instrumental training, an association develops between the instrumental response and its consequent outcome (Bolles, 1972; Colwill \& Rescorla, 1986; Mackintosh \& Dickinson, 1979). One finding is that instrumental performance is affected by postconditioning manipulations of the value of the outcome (Adams \& Dickinson, 1981; Colwill \& Rescorla, 1985). For example, Colwill and Rescorla (1985) found that performance of a response was selectively depressed if its outcome was made less attractive. The other finding is that a discriminative stimulus trained with one response-outcome $(\mathrm{R}-\mathrm{O})$ relation will selectively promote performance of another response trained with the same outcome (Colwill \& Rescorla, 1988a). This transfer test is especially powerful because for an $\mathrm{S}+$ to augment performance of a new response trained with the same outcome but not with a different outcome implies the presence of both stimulus-outcome $(\mathrm{S}-\mathrm{O})$ and $\mathrm{R}-\mathrm{O}$ associations.

This research was supported by National Science Foundation Grants BNS-8915342 and IBN 9407326. I thank Scott Triola for assistance with data collection in Experiment 3. Correspondence should be addressed to R. M. Colwill, Department of Psychology, Brown University, Box 1853, Providence, RI 02912 (e-mail: ruth_colwill@brown.edu).
Subsequently, considerable attention has been focused on identifying the conditions conducive to either the development or the elimination of an $\mathrm{R}-\mathrm{O}$ association. Colwill and Rescorla (1986) summarized the evidence that $\mathrm{R}-\mathrm{O}$ associations occur across a broad range of instrumental training parameters. For example, $\mathrm{R}-\mathrm{O}$ associations were observed to develop despite the insertion of a delay between the response and the outcome, a reduction in the density of reinforcement, and prolonged training of the response with the outcome. Other studies have shown that, once established, $\mathrm{R}-\mathrm{O}$ associations are resistant to several commonly employed decremental treatments (Colwill, 1994; Rescorla, 1991, 1992a, 1993, 1995). It has been reported that $\mathrm{R}-\mathrm{O}$ associations are unaffected by the passage of time (Colwill, 1994) and that extinction treatments such as nonreinforcement of the response (Colwill, 1994; Rescorla, 1991, 1993) or a negative correlation between the response and its outcome (Colwill, 1994; Rescorla, 1992a) do not weaken the R-O association. Furthermore, Rescorla $(1991,1995)$ has shown that training a response with a new outcome does not reduce its association with a previous outcome.

Several experiments have also examined the effect of noncontingent outcomes on acquisition or extinction of an R-O relation. Hammond (1980) and Dickinson and 
Charnock (1985) demonstrated that acquisition of a response is poor if the reinforcer occurs at a high rate in the absence of that response. Adams and Dickinson (1981) found that performance of an instrumental response was depressed following devaluation of its contingent outcome but not after devaluation of a noncontingentoutcome. Colwill and Rescorla (1986) found that the addition of noncontingent outcome presentations selectively disrupted performance of a response earning that outcome relative to a response earning a different outcome. Similarly, Rescorla and Skucy (1969) and Rescorla (1992a) found that the noncontingent delivery of an outcome was an effective procedure for extinguishing performance of an instrumental response trained with that outcome. Of particular interest is Rescorla's (1992a) finding that those noncontingent outcomes did not eliminate the previously established $\mathrm{R}-\mathrm{O}$ association. When an $\mathrm{S}+$ was presented that signaled the noncontingent outcome, the extinguished response trained with that outcome was elevated as much as a nondecremented control response.

A common occurrence in many of these studies of the effect of noncontingent instrumental outcomes was that the delivery of noncontingent outcomes produced drastic reductions in performance of the instrumental response. For example, Rescorla (1992a) reported that the introduction of noncontingent outcomes produced a rapid drop in responding to a rate of about 1.0 response per minute. Such a decline in response rate restricts the opportunity for any prolonged exposure to frequent occurrences of the response and the delivery of noncontingent outcomes. Evidence that responses do not become associated with noncontingentoutcomes or that $\mathrm{R}-\mathrm{O}$ associations are weakened during noncontingent schedules would be especially compelling if responding could be maintained at a relatively high rate during the noncontingent treatment.

The present studies were directed at assessing the effect of response-noncontingent outcomes on extinction of an association between that response and the noncontingent outcome while performance of the response was maintained by a different contingent outcome. In this way, the introduction of noncontingent outcomes did not reduce response frequency and thus more closely approximated the Pavlovian case where conditioned stimulus frequency remains constant during a noncontingent treatment. Experiments 1 and 2 used a transfer test to assess any weakening of the $\mathrm{R}-\mathrm{O}$ association following non- contingent outcome presentations. Experiments 3 and 4 used a transfer test to examine whether the noncontingent treatments used in Experiments 1 and 2 were adequate to establish an association between a response and its noncontingent outcome.

\section{EXPERIMENT 1}

Experiment 1 used the transfer technique to probe for a decrement in the $\mathrm{R}-\mathrm{O}$ association when the outcome was subsequently made noncontingent with respect to the instrumental response. The basic design of Experiment 1 is shown in Figure 1. Two discriminative stimuli (noise and light) were established as signals for the reinforcement of different responses (nosepoke and handle pull) and different outcomes (food pellets and liquid sucrose). Then, two new responses (leverpress and chain pull) were trained. Each response earned both outcomes. After several sessions, one of the outcomes was presented noncontingently with one response, and the other outcome was presented noncontingently with the other response. Transfer tests in which the lever and chain were available with occasional presentations of the noise and light discriminative stimuli were administered after 12 and 24 noncontingent training sessions. The question of interest is whether a discriminative stimulus will be less effective in promoting a response when that $\mathrm{S}+$ signals the response-noncontingent outcome than when it signals the response-contingent outcome. In other words, as depicted in Figure 1, if noncontingent presentations of an outcome weaken its association with the response, $\mathrm{S} 1$ should be less likely to promote R4 than R3 and S2 should be less likely to promote R3 than R4.

\section{Method}

\section{Subjects}

The subjects were 16 experimentally naive male Holtzman-derived Sprague-Dawley rats (Harlan Co.) approximately 100 days old at the start of the experiment. They were housed individually. Water was always available in the home cage. Daily food intake was regulated so that the animals were maintained at $80 \%$ of their freefeeding body weight.

\section{Apparatus}

The apparatus consisted of eight identical Skinner boxes measuring $22.9 \times 20.3 \times 20.3 \mathrm{~cm}$. The two end walls of the chamber were aluminum, and the side walls and ceiling were made of Plexiglas.

\section{S + Training Acquisition Noncontingent Training Transfer Test}

\begin{tabular}{l|l|l|l}
\hline $\mathrm{S} 1: \mathrm{R} 1-\mathrm{O} 1$ & $\mathrm{R} 3-\mathrm{O} 1, \mathrm{R} 3-\mathrm{O} 2$ & $\mathrm{R} 3-\mathrm{O} 1, \mathrm{R} 3-\mathrm{O} 2 / \mathrm{O} 2$ & $\mathrm{~S} 1: \mathrm{R} 3$ v R4 \\
S2: R2-O2 & R4-O1, R4-O2 & R4-O2, R4-O1/O1 & S2: R3 v R4
\end{tabular}

Figure 1. Basic design of Experiment 1: S1 and S2 are instrumental discriminative stimuli-noise and light; $\mathrm{R} 1, \mathbf{R 2}, \mathrm{R3}$, and $\mathrm{R} 4$ are instrumental responses-nosepoking, handle pulling, leverpressing, and chain pulling; $\mathrm{O1}$ and $\mathrm{O2}$ denote food pellets and sucrose liquid. 
The floor of the chamber was composed of $.48-\mathrm{cm}$ stainless steel rods spaced $1.9 \mathrm{~cm}$ apart. Each chamber had a recessed food magazine in the center of one end wall. Sucrose liquid and food pellets were delivered through separate tubes inserted through the roof of the food magazine. Each operation of the sucrose dispenser allowed $0.2 \mathrm{ml}$ of $8 \%$ sucrose to flow onto the floor of the food magazine where it collected in a shallow indentation. Each operation of the pellet dispenser allowed a single 45-mg food pellet (Formula A, P. J. Noyes Co.) to drop onto the floor of the magazine.

Each box was equipped with four manipulanda: a lever, a chain pull, a nosepoke, and a handle pull. The lever was mounted $2.5 \mathrm{~cm}$ from the right-hand wall of the food magazine. The chain was suspended from a microswitch mounted on the roof of the chamber. The end of the chain was $11 \mathrm{~cm}$ from the grid floor and $3 \mathrm{~cm}$ from the left-hand wall of the food magazine. Located $5.5 \mathrm{~cm}$ directly above the roof of the magazine was the nosepoke manipulandum, which consisted of a circular aperture, $2 \mathrm{~cm}$ in diameter and $1.3 \mathrm{~cm}$ deep. The back of this aperture was covered by a metal plate that operated a microswitch whenever it was depressed. Mounted on the same side of the chamber as the chain but $1.5 \mathrm{~cm}$ below the grid floor was the handle pull manipulandum. This consisted of a short flat rod protruding $3 \mathrm{~cm}$ into the chamber. Whenever the rod was pulled upward, a microswitch was closed and a response recorded. The same model of microswitch (Unimax Switch Co., 2HBT-1) was used to detect responding on all four manipulanda. Access to these manipulanda was prevented by covering the lever with a metal plate or by retracting it, by retracting the chain through an opening in the ceiling, by inserting a metal cover into the aperture of the nosepoke manipulandum, and by withdrawing the arm of the handle pull.

Each Skinner box was enclosed in a sound-attenuating and lightresistant shell. A loudspeaker mounted in the corner of the back wall inside the shell permitted presentation of a white noise $(\mathrm{N})$, measuring approximately $76 \mathrm{~dB}$ re $20 \mu \mathrm{N} / \mathrm{m}^{2}$. A 6-W light (L) was mounted on the side wall of each operant chamber about $3 \mathrm{~cm}$ above the grid floor. Experimental events were controlled and recorded automatically by interfacing (Med Associates) and a Zeos 386SX microprocessor located in an adjoining room.

\section{Procedure}

Magazine training. Subjects were given one session of magazine training in which 10 food pellet reinforcers followed by 10 liquid sucrose reinforcers were delivered on a variable-time (VT) 60-sec schedule. No manipulanda were available during this session.

Response training. All subjects were trained to nosepoke and handle pull for pellet and sucrose rewards. Initially, each response was trained on a continuous reinforcement schedule (CRF) until 30 reinforcers had been earned. Eight subjects were trained to nosepoke for pellets in Session 1 and to handle pull for sucrose in Session 2; 8 other subjects were trained to nosepoke for sucrose in Session 1 and to handle pull for pellets in Session 2.

Following CRF training, subjects received two 20-min sessions with each response reinforced on a variable-interval (VI) 30-sec schedule. The order of training was as follows: nosepoke, handle pull, handle pull, and nosepoke. Responses earned the same outcomes as they had in CRF training.

Discriminative stimulus training. Subjects were trained to respond (nosepoke or handle pull) in the presence of an S+ (noise or light discriminative stimulus) for a rewarding outcome (food pellet or liquid sucrose). Each training session contained one of the response manipulanda and 32 presentations of either a 30-sec noise or light S+. Responses during an S+ were reinforced on a VI 30-sec schedule, but responses were never reinforced during the intertrial interval (ITI). For 8 subjects, nosepoking was reinforced in the light $\mathrm{S}+$ and handle pulling in the noise $\mathrm{S}+$; for the remaining 8 subjects, these stimulus-response ( $\mathrm{S}-\mathrm{R})$ combinations were switched. Within each of these conditions, $\mathrm{R}-\mathrm{O}$ combinations were counterbalanced; thus, 4 subjects received pellets for nosepoking and sucrose for handle pulling, and 4 subjects received sucrose for nosepoking and pellets for handle pulling. In this way, each subject was trained with one stimulus as an $\mathrm{S}+$ for one $\mathrm{R}-\mathrm{O}$ relation and with another stimulus as an $\mathrm{S}+$ for a different $\mathrm{R}-\mathrm{O}$ relation. There were 10 sessions of $\mathrm{S}+$ training with each response. The ITI was gradually increased over sessions: The mean ITI was $15 \mathrm{sec}$ in the first session, $30 \mathrm{sec}$ in the next three sessions, $60 \mathrm{sec}$ in the following three sessions, and $90 \mathrm{sec}$ in the final three sessions, for each response.

Transfer response training. Two new responses, leverpressing and chain pulling, were trained with both sucrose and pellets. Initially, each response was given one session of CRF training with each outcome until 30 reinforcers had been earned. The order of training was lever-pellets, chain-pellets, lever-sucrose, and chain-sucrose. Subjects were then given two 20-min sessions of VI 30-sec training with each response. Responses earned sucrose in the first session and pellets in the second session. All animals then received five 20min sessions with each response earning both pellets and sucrose on independent VI 60-sec schedules with the constraint that a reinforcer that had been set up had to be collected before any other reinforcer could be set up.

All animals then received twenty-four 20-min sessions with each response. In each session, a response continued to earn both outcomes on VI 60 -sec schedules, but one of the outcomes was also presented on a VT 60 -sec schedule. The identity of the noncontingent outcome was different for each response. In this way, the design matched overall exposure to and treatment of both outcomes: For half the animals, the sucrose outcome was contingent on leverpressing and noncontingent on chain pulling, and the pellet outcome was noncontingent on leverpressing and contingent on chain pulling; for the remaining animals, the pellet outcome was contingent on leverpressing and noncontingent on chain pulling, and the sucrose outcome was noncontingent on leverpressing and contingent on chain pulling.

A transfer test was administered prior to the start of the noncontingent treatment, after 12 sessions of noncontingent training with each response, and finally after a total of 24 sessions of noncontingent training with each response.

Transfer tests. Each transfer test was preceded by $\mathrm{S}+$ retraining. The training parameters were the same as those in effect for the final sessions of $S+$ training except that each $S+$ was trained for only half a session. In the first transfer test, both the lever and chain were available, but responding was never reinforced. After a 4-min extinction period, there were four presentations of each 30 -sec S+ with a mean 30 -sec ITI. The order of stimulus presentations was NLLNLNNL. For the transfer tests administered after 12 and 24 sessions of noncontingent training, both the lever and chain were available, but responding was never reinforced. There were eight presentations of each 30-sec S+ with a mean 30-sec ITI. The order of stimulus presentations was NLLNLNNL for the first eight trials. The sequence was reversed for the last eight trials.

\section{Results and Discussion \\ Discriminative Stimulus Training}

All subjects acquired the initial $\mathrm{S}+$ discrimination. By the end of training, the rate of responding was substantially higher during the noise and light $\mathrm{S}+\mathrm{s}$ (34.2 responses per $\mathrm{min}$ ) than during the ITI ( 3.6 responses per min). The mean discrimination ratio for the final session collapsed across stimulus, response and outcome identity was .91. Discriminative performance was consistent across the retraining sessions that preceded each of the three transfer tests. Mean discrimination ratios in the half sessions prior to the transfer tests were $.88, .86$, and .85 , respectively. 


\section{Transfer Response Training}

All subjects learned to leverpress and chain pull. The mean response rates in the final training sessions preceding each of the transfer tests were 10.9 (final training session before noncontingent treatment), 13.3 (Session 12 of noncontingenttreatment), and 13.8 (Session 24 of noncontingent treatment) chain pulls per min and 14.6 (final training session before noncontingent treatment), 18.0 (Session 12 of noncontingent treatment), and 18.0 (Session 24 of noncontingent treatment) leverpresses per min.

\section{Transfer Tests}

The results of the first transfer test indicated that the $\mathrm{S}+\mathrm{s}$ elevated performance of both responses relative to the ITI. The mean rate of responding during the stimuli (17.1 responses per min) was significantly higher than the mean ITI rate (15.0 responses per $\min )[T(15)=20$, $p<.05]$. These results indicate the success of S+ training and initial transfer response training in establishing $\mathrm{S}-\mathrm{O}$ and $\mathrm{R}-\mathrm{O}$ associations, respectively. Moreover, they demonstrate that an $\mathrm{S}+$ can simultaneously promote two responses with which it shares an outcome.

The data of most interest come from the two transfer tests administered after 12 and 24 sessions of noncontingent training. These data are plotted in Figure 2. Mean responses per minute are shown separately during $\mathrm{S}+$ presentations when the outcome signaled by $\mathrm{S}+$ was the same as the response-contingentoutcome $(\mathrm{C})$ and when it was the same as the response-noncontingent outcome
(NC). Response rates are also displayed during the ITI when no stimuli were presented. In each transfer test, an $\mathrm{S}+$ promoted performance of both the contingent and noncontingent responses.

Analysis of these data revealed that there was no significant difference in the effect of an $\mathrm{S}+$ on its contingent or noncontingentresponse in either transfer test $[T(16)=$ 48.5 , n.s., $T(16)=64$, n.s., respectively]. In the first test, an S+ significantly increased performance of the contingent response relative to the ITI [T $(16)=26, p<.05]$, and the noncontingent response relative to the ITI $[T(16)=$ $18, p<.01]$. These differences between the stimulus rates and the ITI rate were still apparent in the second test but were marginally nonsignificant $[T \mathrm{~s}(15)=28.5, p \mathrm{~s}>.05]$. It seems likely that repeated testing of the discriminative stimuli with the transfer responses followed by retraining of the responses in the absence of the S+s may have resulted in their differential extinction during the stimuli, which in turn contributed to the difficulty of detecting significant elevation of performance by $\mathrm{S}+$ relative to the ITI.

These data provide no evidence that exposing a response to noncontingent presentations of its outcome reduces the strength of the $\mathrm{R}-\mathrm{O}$ association even when performance of that response is maintained during the decremental operation. However, one might be concerned that the continued availability of the noncontingent outcome on a VI schedule may have been sufficient to counteract any decremental effect of its delivery on a VT schedule. A more

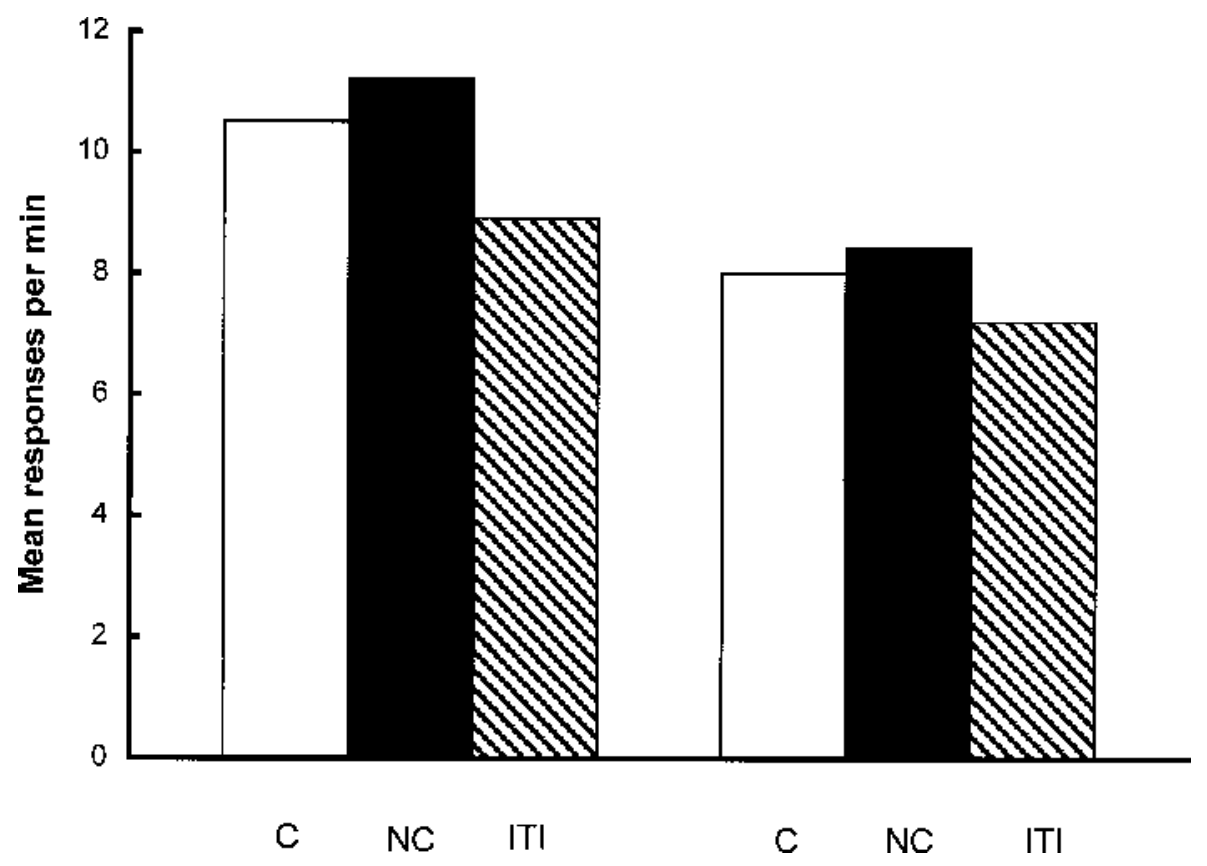

Figure 2. Experiment 1: Results of transfer tests of discriminative stimuli $(\mathrm{S}+\mathrm{s})$ and instrumental responses given 12 (Test 1, left) or 24 (Test 2 , right) noncontingent extinction sessions. Responding is shown during a stimulus that signaled an outcome that was contingent on the response $(\mathrm{C})$, during a stimulus that signaled an outcome that was noncontingent on the response (NC), and during the intertrial interval (ITI), when no stimuli were present. 
stringent test of the effect of a noncontingent outcome on a previously established $\mathrm{R}-\mathrm{O}$ association would be to make all presentations of that outcome noncontingent while maintaining performance of the response with a different outcome. This procedure was used in the next experiment.

\section{EXPERIMENT 2}

Experiment 2 examined further the effect of a noncontingent outcome on a previously established association between that outcome and an instrumental response. The basic design of this experiment is shown in Figure 3. As in Experiment 1, each subject received S+ training with two discriminative stimuli (noise and light), two responses (nosepoke and handle pull), and two outcomes (food pellets and sucrose liquid). Then, two new responses (leverpress and chain pull) were trained with the two outcomes. Finally, each response continued to earn one outcome while the other outcome was delivered independently of responding. Each outcome was contingenton one response and noncontingent on the other response so that overall exposure and treatment of both outcomes was matched.

Transfer tests were carried out to assess the impact of the noncontingent outcome presentations on $\mathrm{R}-\mathrm{O}$ associations. A choice test was conducted after each response had been separately tested in the presence of the noise and light $\mathrm{S}+\mathrm{s}$. It was expected that if noncontingent presentations of an outcome weakened the $\mathrm{R}-\mathrm{O}$ association, an $\mathrm{S}+$ that had signaled that outcome would be less able to elevate performance of that response. In other words, during S1 presentations, performance of R4 should be less likely than performance of R3; but during S2 presentations, performance of R3 should be less likely than performance of R4.

\section{Method}

\section{Subjects and Apparatus}

Sixteen experimentally naive male Holtzman-derived SpragueDawley rats served as subjects. They were about 100 days old at the start of the experiment and were housed and maintained under the same conditions as subjects in Experiment 1. The apparatus used was the same as that employed in Experiment 1.

\section{Procedure}

Magazine training. The procedure for magazine training was the same as that used in Experiment 1.
Response and $\mathbf{S}+$ training. The procedure for training subjects to nosepoke and handle pull in the presence of discriminative stimuli was identical to that of Experiment 1.

Transfer response training. The procedure for initially training two new responses, leverpressing and chain pulling, with contingent outcomes was the same as that used in Experiment 1. The procedure differed from that of Experiment 1 with regard to the arrangement of the noncontingent outcome during the decremental phase. All animals received twenty-four 20-min sessions with each response. In each session, a response continued to earn one outcome on VI 60-sec schedule, but the other outcome was presented only on a VT 60 -sec schedule. Thus, each outcome was positively correlated with one response and delivered noncontingently with respect to the other response. In this way, the design matched overall exposure to and treatment of both outcomes: For half the animals, the sucrose outcome was contingent on leverpressing and noncontingent on chain pulling, and the pellet outcome was noncontingent on leverpressing and contingent on chain pulling; for the remaining animals, the pellet outcome was contingent on leverpressing and noncontingent on chain pulling, and the sucrose outcome was noncontingent on leverpressing and contingent on chain pulling.

Transfer tests. Single response transfer tests were administered after a total of 24 sessions with each response on the noncontingent schedule. The tests were preceded by $\mathrm{S}+$ retraining. The training parameters were the same as those in effect for the final sessions of $\mathrm{S}+$ training. There were four presentations of each $30-\sec S+$ with a mean 30 -sec ITI. The order of stimulus presentations was NLLNLNNL. The lever was available in the first test session, and the chain was available in the second test session.

A choice test was given the following day. Both response manipulanda were available in this test with intermittent presentations of the $30-\mathrm{sec}$ light and noise $\mathrm{S}+\mathrm{s}$. There were eight presentations of each stimulus with a mean ITI of $30 \mathrm{sec}$. The order of stimulus presentations was NLLNLNNLLNNLNLLN.

\section{Results and Discussion}

\section{Discriminative Stimulus Training}

All subjects acquired the initial $\mathrm{S}+$ discrimination. By the end of training, the rate of responding was substantially higher during the noise and light $\mathrm{S}+\mathrm{s}$ (31.0 responses per min) than during the ITI (4.1 responses per min). The mean discrimination ratio for the final session collapsed across stimulus, response, and outcome identity was .89. Discriminative performance remained at a satisfactory level, and the mean discrimination ratio in the half sessions prior to testing was .85 .

\section{Transfer Response Training}

All subjects learned to leverpress and chain pull. In the final training sessions immediately preceding intro-

\section{S+ Training Acquisition Noncontingent Training Transfer Test}

\begin{tabular}{l|l|l|l}
\hline S1: R1-O1 & R3-O1, R3-O2 & R3-O1, O2 & S1: R3, S2: R4 \\
S2: R2-O2 & R4-O1, R4-O2 & R4-O2, O1 & S1: R4, S2: R3
\end{tabular}

Figure 3. Basic design of Experiment 2: S1 and S2 are instrumental discriminative stimuli-noise and light; $R 1, R 2, R 3$, and $\mathbf{R} 4$ are instrumental responses-nosepoking, handle pulling, leverpressing, and chain pulling; $\mathrm{O1}$ and $\mathrm{O} 2$ denote food pellets and sucrose liquid. 
duction of the noncontingent treatment, the mean rate of chain pulling was 12.1 responses per min, and the mean rate of leverpressing was 12.9 responses per min. The mean response rates in the final training sessions with noncontingent outcomes that immediately preceded each of the transfer tests were 10.1 chain pulls per min and 10.7 leverpresses per min.

\section{Transfer Tests}

The results of most interest come from the single response transfer tests and are summarized in Figure 4. Mean responses per minute are shown separately during $\mathrm{S}+$ presentations where $\mathrm{S}+$ signaled the outcome that was positively correlated with the response and where S+ signaled the outcome that was noncontingent on the response. Response rates are also displayed during the ITI when no stimuli were presented. Inspection of Figure 4 reveals that responding was promoted equally by an $\mathrm{S}+$ that signaled the contingent outcome and by an $\mathrm{S}+$ that signaled the noncontingent outcome. Statistical analysis of these data revealed that there was no significant difference in the effect of an $\mathrm{S}+$ on the contingent response or on the noncontingent response $[T(16)=65$, n.s. $]$. An $\mathrm{S}+$ significantly increased performance of both the positively correlated response relative to the ITI $[T(16)=3$, $p<.01]$ and the noncontingent response relative to the ITI $[T(16)=12, p<.01]$.

The pattern of data shown in Figure 4 was reproduced in essence in the choice transfer test. There was no difference in the rate of responding during an $\mathrm{S}+$ that signaled either the contingent (6.2 responses per min) or noncontingent outcome $(6.0$ responses per $\min )[T(16)=60$, n.s.]. However, responding in both cases was elevated relative to the ITI (5.2 responses per $\min )[T \mathrm{~s}(16) \leq 30$, $p$ s $\leq .05]$.

These data suggest that exposing a response to noncontingent presentations of its outcome neither eliminates nor weakens a previously established association between that response and the outcome even when performance of that response is maintained by another outcome during the decremental operation. The results are in agreement with those reported by Rescorla (1992a), who found preservation of an $\mathrm{R}-\mathrm{O}$ association despite elimination of performance of that response by noncontingent presentations of its outcome.

\section{EXPERIMENT 3}

One reason for the failure of the noncontingent schedules used in Experiments 1 and 2 to weaken preexisting $\mathrm{R}-\mathrm{O}$ associations is that the high rate of responding maintained by the contingent outcome may have resulted in frequent contiguities between that response and its noncontingent outcome. The next two experiments evaluated whether these potential contiguities were sufficient to support the development of an association between a response and its noncontingentoutcome. As in Experiments 1 and 2, rats were first trained to nosepoke or handle pull for food pellet or sucrose rewards in the presence of noise or light discriminative stimuli. One response (R1) earned one outcome (O1) during presentations of one stimulus (S1); the other response (R2) earned another outcome (O2) during presentations of another stimulus (S2). Then, two new responses were established; one of these re-

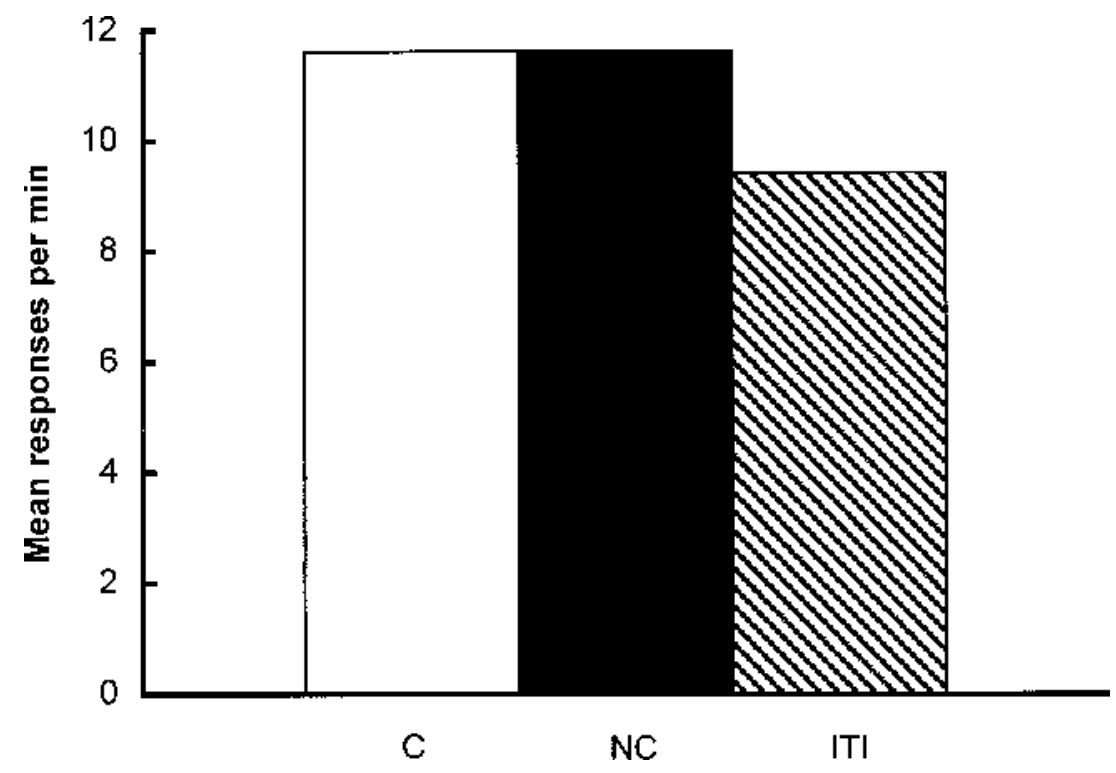

Figure 4. Experiment 2: Results of single-response transfer tests of discriminative stimuli $(\mathrm{S}+\mathrm{s})$ and instrumental responses given 24 noncontingent extinction sessions. Responding is shown during a stimulus that signaled an outcome that was contingent on the response $(\mathrm{C})$, during a stimulus that signaled an outcome that was noncontingent on the response (NC), and during the intertrial interval (ITI), when no stimuli were present. 
sponses (R3) earned O1 and the other response (R4) earned $\mathrm{O} 2$. After a brief amount of training, each response continued to earn its original outcome on a VI 60-sec schedule but was also exposed to noncontingent presentations of the other outcome. In Experiment 3, this noncontingent procedure was the same as that used in Experiment 1 . Thus, the noncontingent outcome was presented on a VI 60-sec schedule and on a VT 60-sec schedule. Transfer tests were conducted with R3 and R4 and the two discriminative stimuli, S1 and S2. It was expected that performance of a response would be promoted by an $\mathrm{S}+$ that signaled the contingent outcome but not by an S+ that signaled the noncontingent outcome. Thus, $\mathrm{S} 1$ should promote R3 but not R4, and S2 should promote R4 but not $\mathrm{R} 3$. Evidence that $\mathrm{S} 1$ promotes $\mathrm{R} 4$ and that $\mathrm{S} 2$ promotes $\mathrm{R} 3$ would suggest the acquisition of an $\mathrm{R}-\mathrm{O}$ association even when $\mathrm{O}$ was noncontingent. The basic design is outlined in Figure 5.

\section{Method}

\section{Subjects and Apparatus}

Sixteen experimentally naive male Holtzman-derived SpragueDawley rats served as subjects. They were housed and maintained under the same conditions as subjects in Experiment 1. The apparatus used was the same as that employed in Experiment 1.

\section{Procedure}

Magazine training. The procedure for magazine training was the same as that used in Experiment 1.

Response and $\mathbf{S}+$ training. The procedure for training subjects to nosepoke and handle pull in the presence of discriminative stimuli was identical to that of Experiment 1.

Transfer response training. Two new responses, leverpressing and chain pulling, were trained. Each response was trained initially on a CRF schedule until 30 reinforcers had been earned. Subjects were then given one 20-min session of training with each response in which its outcome was available on a VI 30-sec schedule, and four 20-min sessions with each response reinforced on a VI 60-sec schedule. For half the animals, pellets served as the reinforcer for leverpressing and sucrose for chain pulling. For the remaining animals, these $\mathrm{R}-\mathrm{O}$ combinations were switched. These assignments were counterbalanced with respect to $S+$ training.

All animals then received twenty-four 20-min sessions with each response. In each session, a response continued to earn its original training outcome on a VI 60-sec schedule. The other outcome was presented on a VI 60-sec schedule and on a VT 60-sec schedule. In this way, each outcome was contingent on one response but occurred noncontingently with another response. This design matched overall exposure to and treatment of both outcomes: For half the animals, the sucrose outcome was contingent on leverpressing and non- contingent on chain pulling, and the pellet outcome was noncontingent on leverpressing and contingent on chain pulling; for the remaining animals, the pellet outcome was contingent on leverpressing and noncontingent on chain pulling, and the sucrose outcome was noncontingent on leverpressing and contingent on chain pulling. A transfer test was administered after 12 sessions with each response and after a total of 24 sessions with each response.

Transfer tests. Prior to testing the discriminative stimuli with the transfer responses, each $\mathrm{S}+$ was retrained with its original response for half a session. The training parameters were the same as those in effect for the final sessions of S+ training. In each of the two transfer test sessions, both the lever and chain were available, but responding was never reinforced. In each test, there were eight 30 -sec presentations of each $\mathrm{S}+$ with a mean 30 -sec ITI. The order of stimulus presentations was NLLNLNNL for the first eight trials. The sequence was reversed for the last eight trials.

\section{Results and Discussion \\ Discriminative Stimulus Training}

All subjects acquired the initial $\mathrm{S}+$ discrimination. By the end of training, the rate of responding was substantially higher during the noise and light $\mathrm{S}+\mathrm{s}$ (25.3 responses per $\min$ ) than during the ITI (4.1 responses per min). The mean discrimination ratio for the final session collapsed across stimulus, response, and outcome identity was .86. Discriminative performance was consistent across the retraining sessions that preceded each of the two transfer tests. Mean discrimination ratios in the half sessions prior to the transfer tests were .80 and .82 , respectively.

\section{Transfer Response Training}

All subjects learned to leverpress and chain pull. In the final sessions preceding introduction of the noncontingent treatment, the mean rate of chain pulling was 7.6 responses per min and the mean rate of leverpressing was 12.0 responses per min. The mean response rates in the final training sessions preceding each transfer test were 11.0 (Session 12 of noncontingenttraining) and 12.1 (Session 24 of noncontingent training) chain pulls per min and 14.1 (Session 12 of noncontingenttraining) and 14.9 (Session 24 of noncontingent training) leverpresses per min.

\section{Transfer Tests}

The data of most interest come from the two transfer tests and are summarized in Figure 6. Mean responses per min are shown separately during an $\mathrm{S}+$ trained with the outcome that was contingent on the response (C), during an S+ trained with the outcome that was noncon-

\section{S+ Training Acquisition Noncontingent Training Transfer Test}

\begin{tabular}{l|l|l|l}
\hline S1: R1-O1 & R3-O1 & R3-O1, R3-O2/O2 & S1: R3 v R4 \\
S2: R2-O2 & R4-O2 & R4-O2, R4-O1/O1 & S2: R3 v R4
\end{tabular}

Figure 5. Basic design of Experiment 3: S1 and S2 are instrumental discriminative stimuli-noise and light; $\mathbf{R 1}, \mathbf{R 2}, \mathbf{R 3}$, and $\mathbf{R} 4$ are instrumental responses-nosepoking, handle pulling, leverpressing, and chain pulling; $\mathrm{O} 1$ and $\mathrm{O} 2$ denote food pellets and sucrose liquid. 


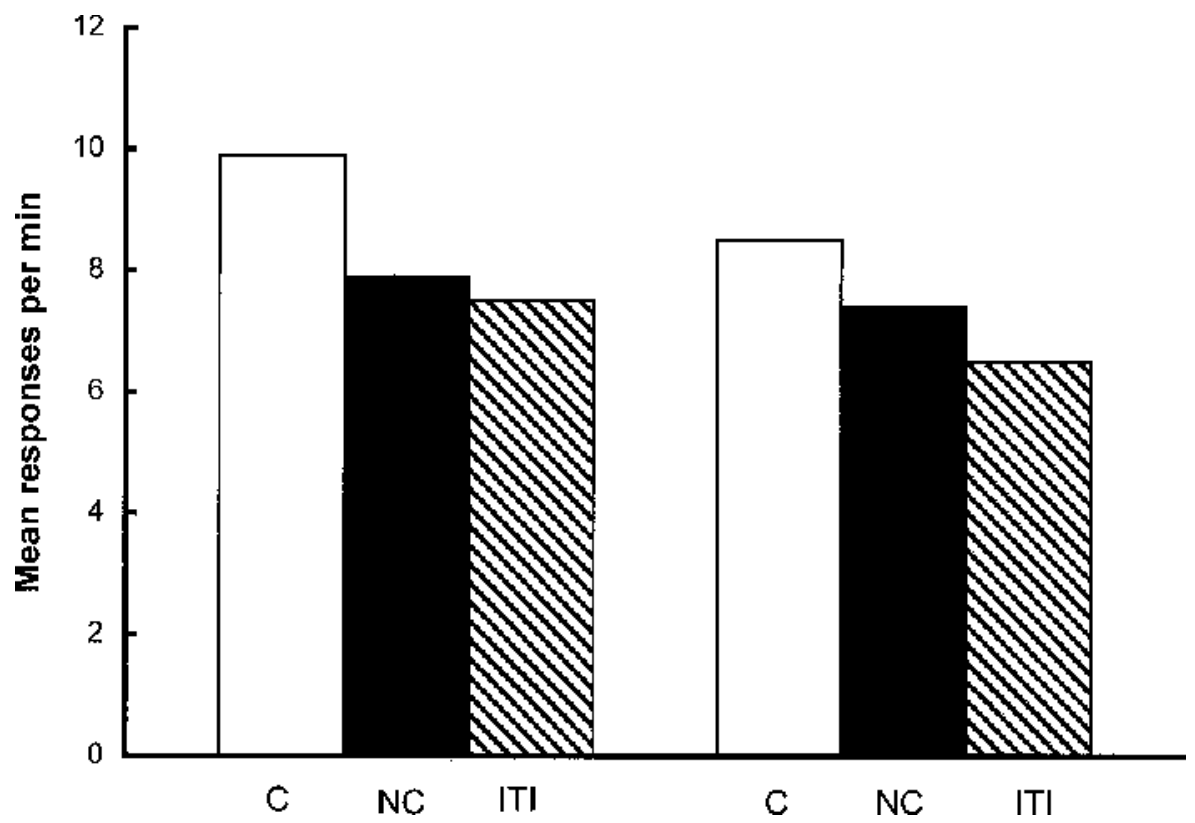

Figure 6. Experiment 3: Results of transfer tests of discriminative stimuli $(\mathrm{S}+\mathrm{s})$ and instrumental responses given 12 (Test 1 , left) or 24 (Test 2 , right) noncontingent acquisition sessions. Responding is shown during a stimulus that signaled an outcome that was contingent on a response (C), during a stimulus that signaled an outcome that was noncontingent on a response (NC), and during the intertrial interval (ITI) when no stimuli were present.

tingent on the response (NC), and in the absence of any $\mathrm{S}+$ (ITI). In each transfer test, it is clear that an $\mathrm{S}+\mathrm{se}-$ lectively promoted performance of the contingent response but only marginally increased performance of the noncontingent response.

Statistical analysis of the data from the first transfer test revealed that responding was significantly higher in an $\mathrm{S}+$ that signaled the contingent outcome than in an $\mathrm{S}+$ that signaled the noncontingent outcome [Wilcoxon $T(16)=$ $15, p<.01]$. Relative to the ITI, $\mathrm{S}+$ increased performance of the contingent response $[T(16)=4, p<.01]$ but had no statistically significant effect on the noncontingent response $[T(16)=51.5$, n.s.]. Similar findings were obtained in the second transfer test. During S+ presentations, responding was significantly higher in the contingent condition than in the noncontingent condition [Wilcoxon $T(15)=20.5, p<.05]$. Relative to the ITI, the contingent response was elevated by an $\mathrm{S}+[T(16)=13, p<.01]$, but the increase in the noncontingent response was negligible $[T(16)=35$, n.s.].

These results show that despite considerable exposure to noncontingent presentations of an outcome, performance of an instrumental response was not enhanced by an S+ that signaled that outcome. Thus, despite a substantial number of contiguities between the response and the noncontingent outcome, the additional responseindependent presentations of that outcome were apparently sufficient to prevent the formation of a strong $\mathrm{R}-\mathrm{O}$ association. This finding is consistent with the now com- monly accepted contemporary view that an event has to be contingent with an outcome to become associated with that outcome.

\section{EXPERIMENT 4}

The issue of whether the noncontingentoutcome schedule used in Experiment 2 might be sufficient to produce an association between that outcome and its instrumental response was examined in Experiment 4. The design of this experiment is shown in Figure 7. Discriminative stimuli were first established as signals for different $\mathrm{R}-\mathrm{O}$ associations. Then, two transfer responses were trained. Each response had a contingent outcome and a noncontingent outcome. This procedure differed from that of Experiment 3 in that for each response, one outcome was scheduled on a VI 60 -sec schedule and the other outcome was scheduled on a VT 60-sec schedule. Thus, as in Experiment 2, there were no explicitly arranged contiguities between a response and its noncontingent outcome. Finally, single response transfer tests were conducted to detect the presence of an association, if any, between a response and its noncontingent outcome. It was expected that $\mathrm{S} 1$ and $\mathrm{S} 2$ would promote performance of R3 and $\mathrm{R} 4$, respectively. The question of interest was whether S1 and S2 would also promote performance of R4 and R3, respectively. Significant elevation of these responses relative to the ITI would suggest that $\mathrm{R}-\mathrm{O}$ associations do develop between responses and their noncontingent outcomes. 


\begin{tabular}{l|c|c|c} 
S+ Training & Acquisition & Noncontingent Training & Transfer Test \\
S1: R1-O1 & R3-O1 & R3-O1, O2 & S1: R3, S2: R4 \\
S2: R2-O2 & R4-O2 & R4-O2, O1 & S1: R4, S2: R3
\end{tabular}

\begin{abstract}
Figure 7. Basic design of Experiment 4: S1 and S2 are instrumental discriminative stimuli-noise and light; $\mathrm{R} 1, \mathrm{R} 2, \mathrm{R3}$, and $\mathrm{R} 4$ are instrumental responses-nosepoking, handle pulling, leverpressing, and chain pulling; $\mathrm{O} 1$ and $\mathrm{O} 2$ denote food pellets and sucrose liquid.
\end{abstract}

\begin{abstract}
Method

\section{Subjects and Apparatus}

Sixteen experimentally naive 100-day-old male Holtzmanderived Sprague-Dawley rats served as subjects. They were housed and maintained under the same conditions as subjects in Experiment 1 . The apparatus used was the same as that employed in Experiment 1.
\end{abstract}

\section{Procedure}

Magazine training. The procedure for magazine training was the same as that used in Experiment 1.

Response and $\mathbf{S}+$ training. The procedure for training subjects to nosepoke and handle pull in the presence of discriminative stimuli was identical to that of Experiment 1.

Transfer response training. The procedure used to train two new responses, leverpressing and chain pulling, was identical to that of Experiment 3. Following the completion of four sessions of VI 60-sec training with each response, the noncontingent outcome was introduced. In each session, a response continued to earn its original training outcome on a VI 60 -sec schedule while the other outcome was presented on a VT 60 -sec schedule. In this way, each outcome was contingent on one response but not contingent on the other response. This design matched overall exposure to and treatment of both outcomes: For half the animals, the sucrose outcome was contingent on leverpressing and noncontingent on chain pulling, and the pellet outcome was noncontingent on leverpressing and contingent on chain pulling; for the remaining animals, the pellet outcome was contingent on leverpressing and noncontingent on chain pulling, and the sucrose outcome was noncontingent on leverpressing and contingent on chain pulling. All animals received twenty-four 20min training sessions with each response.

Transfer testing. Prior to testing the discriminative stimuli with the transfer responses, each $\mathrm{S}+$ was retrained with its original response for half a session. The training parameters were the same as those in effect for the final sessions of $\mathrm{S}+$ training. A transfer test session contained four $30-\mathrm{sec}$ presentations of each $\mathrm{S}+$ with a mean 30-sec ITI. The order of stimulus presentations was NLLNLNNL. The chain was available in the first test session and the lever was available in the second test session. Responding was never reinforced during these tests.

\section{Results and Discussion}

\section{Discriminative stimulus training}

All subjects acquired the initial $\mathrm{S}+$ discrimination. By the end of training, the rate of responding was substantially higher during the noise and light $\mathrm{S}+\mathrm{s}$ (36.6 responses per min) than during the ITI ( 3.9 responses per min). The mean discrimination ratio for the final session collapsed across stimulus, response, and outcome identity was .90 .
The mean discrimination ratio in the half sessions prior to testing was .84 .

\section{Transfer Response Training}

All subjects learned to leverpress and chain pull. The mean rate of chain pulling was 8.6 responses per min, and the mean rate of leverpressing was 13.6 responses per $\min$ in the final session of contingent-outcome training prior to the introduction of the noncontingent treatment. The mean response rates in the final training sessions with the noncontingent outcome that immediately preceded transfer testing were 10.0 chain pulls per min and 10.9 leverpresses per min.

\section{Transfer Tests}

Figure 8 plots the data from the transfer test. Mean responses per min are shown separately during an $\mathrm{S}+$ trained with the same outcome as that made contingent on the response $(\mathrm{C})$, during an $\mathrm{S}+$ trained with the same outcome as that made noncontingent on the response (NC), and in the absence of any S+ (ITI). It is clear that an S+ had a substantially larger effect on performance of the contingent response than on the noncontingent response. Statistical analysis of the data revealed that responding was significantly higher in an S+ that signaled the contingent outcome than in an $\mathrm{S}+$ that signaled the noncontingent outcome [Wilcoxon $T(16)=8.5, p<.01$ ]. Relative to the ITI, however, performance of both the contingent and noncontingent responses was significantly increased $[T(16)=0, p<.01$ and $T(16)=7, p<.01$, respectively $]$.

The overall pattern of responding during the singleresponse transfer tests was similar to that obtained in the choice transfer tests in Experiment 3. However, in Experiment 4 , the slightly elevated level of performance of a response during an $\mathrm{S}+$ that signaled its noncontingent outcome relative to the ITI was statistically significant. One possible reason for this difference between the experiments is that a weak association did in fact develop between the response and its noncontingent outcome in Experiment 3, but its detection was obscured because of competition from the concurrently available contingent response. The presence of a weak association between a response and its noncontingentoutcome has been reported elsewhere (Rescorla, 1992a). Analyses of the source of 


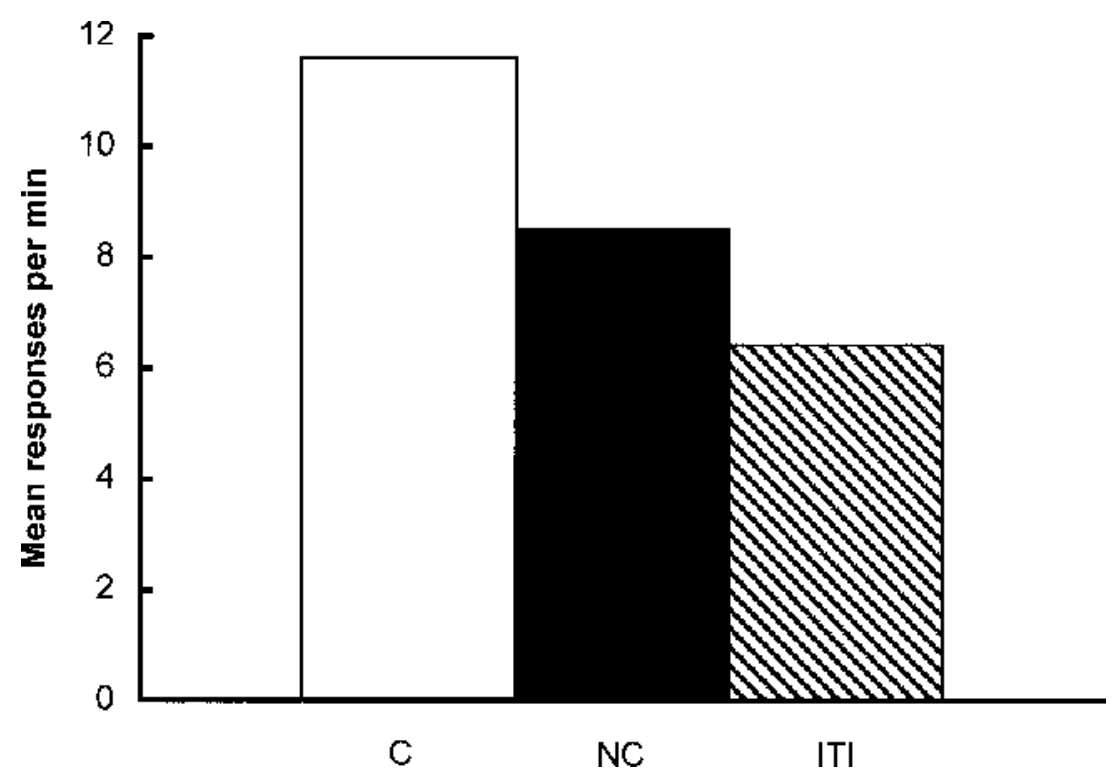

Figure 8. Experiment 4: Results of single response transfer tests of discriminative stimuli $(\mathrm{S}+\mathrm{s})$ and instrumental responses given 24 noncontingent acquisition sessions. Responding is shown during a stimulus that signaled an outcome that was contingent on the response $(C)$, during a stimulus that signaled an outcome that was noncontingent on the response (NC), and during the intertrial interval (ITI) when no stimuli were present.

excitatory Pavlovian conditioning in random control procedures would suggest that the weak $\mathrm{R}-\mathrm{O}$ association is the result of initial spurious $\mathrm{R}-\mathrm{O}$ contiguities that precede substantial experience with the noncontingent schedule, thereby effectively arranging a brief positive contingency between R and O (Ayres, Benedict, \& Witcher, 1975; Benedict \& Ayres, 1972). The persistence of these marginal associations through the noncontingent treatments is, of course, consistent with the results of Experiments 1 and 2.

\section{GENERAL DISCUSSION}

The main results of these transfer experiments confirm and extend previous findings that the strength of a previously established $\mathrm{R}-\mathrm{O}$ association is not reduced by noncontingent presentations of $\mathrm{O}$. In contrast to previous studies that subjected a response to noncontingent outcome presentations that substantially depressed its performance, Experiments 1 and 2 arranged for responding to be maintained by a different outcome during the decremental treatment. However, despite the increased opportunity for experiencing the noncontingentrelation between the response and its previously earned outcome, there was no evidence that noncontingent outcomes weakened the $\mathrm{R}-\mathrm{O}$ association. In Experiments 1 and 2, a response that had been reinforced with two outcomes remained equally well associated with both of those outcomes even after prolonged exposure to noncontingent presentations of one of those outcomes.

These findings are also consistent with other reports about the persistence of $\mathrm{R}-\mathrm{O}$ associations after perfor- mance of $\mathrm{R}$ has been eliminated either by withholding presentations of $\mathrm{O}$ or by negatively correlating $\mathrm{R}$ and $\mathrm{O}$ (Colwill, 1994; Rescorla, 1991, 1992a, 1993). Colwill (1994) summarized three popular accounts that help explain why original learning remains intact despite the loss of performance produced by these other extinction procedures. One explanation attributes the decline in $\mathrm{R}$ to an increase in a competing behavior. Another explanation assumes that $\mathrm{R}$ is punished by the aversive consequences of an omitted expected reward (Amsel, 1958; Wagner, 1966). A third possibility is that $R$ is suppressed by an inhibitory association that develops between the decremental context and $\mathrm{R}$, just as an inhibitory $\mathrm{S}-\mathrm{R}$ association develops between an $\mathrm{S}$ and its nonreinforced response (Colwill, 1991). Each of these accounts attributes the preservation of the $\mathrm{R}-\mathrm{O}$ association to the protection afforded by a process that produces a rapid decrement in performance of R, thereby keeping its original associative value intact. Consequently, none of these accounts would anticipate preservation of the $\mathrm{R}-\mathrm{O}$ association found in Experiments 1 and 2. Because a high level of responding was maintained with a different contingent outcome, there should have been some adjustment in the strength of the association between the response and its noncontingent outcome.

A general concern that might be raised about the studies that have failed to find any weakening of the $\mathrm{R}-\mathrm{O}$ association following exposure to decremental operations is that the methods (transfer test and outcome devaluation) used to measure $\mathrm{R}-\mathrm{O}$ associative strength lack sufficient sensitivity to detect variations in $\mathrm{R}-\mathrm{O}$ strength. For example, it might be argued that an $\mathrm{S}+$ would promote 
equally any response associated with the same outcome regardless of the strength of that association. The results of Experiment 4 showing superior augmentation by an $\mathrm{S}+$ of the contingent response relative to the noncontingent response suggest that this view, at least in an extreme form, is not accurate. However, the precision with which the transfer test can detect variations in $\mathrm{R}-\mathrm{O}$ strength remains to be fully examined. On the other hand, there is good evidence that the outcome devaluation technique is quite sensitive to differences in the strength of $\mathrm{R}-\mathrm{O}$ associations (Colwill \& Rescorla, 1988b). Yet use of that technique has not yielded any evidence of associative loss following noncontingent or negative correlations of the response and its previously earned outcome or simple extinction of the response (Colwill, 1994; Rescorla, 1991, 1992a, 1993). It seems unlikely, then, that the failure of Experiments 1 or 2 to find that an $\mathrm{S}+$ produced even numerically less elevation of the noncontingent response relative to the contingent response should be attributed to the use of an insensitive assessment method.

A straightforward explanation of the results of Experiments 1 and 2 is that because a different contingent outcome was used to ensure that responding occurred frequently, there were many contiguities or approximate contiguities between the response and the noncontingent outcome. According to this account, the noncontingent outcome actually bore a strong positive contingency to the response and thus sustained its previously established association with the response. However, the results of Experiments 3 and 4 do not provide much support for this analysis. In neither study was there evidence that the noncontingent reinforcement schedules were able to establish any more than a negligible association between the response and the noncontingent outcome. In particular, Experiment 3 found no significant enhancement of responding during an $\mathrm{S}+$ that signaled the response's noncontingent outcome even though at least half of the presentations of that outcome were contiguous with the response. While it is not unheard of for schedules of reinforcement that are ineffective in establishing a new behavior to be effective in maintaining performance of an acquired behavior (E. R. Davis \& Platt, 1983; Rescorla, 1989, 1992a), it should be noted that the noncontingent procedures used in Experiments 1 and 2 are unlikely to have supported performance of the response in the absence of the contingent delivery of the other outcome (Colwill, 1994). Of course, it is possible that an $\mathrm{R}-\mathrm{O}$ association could be maintained by a reinforcement schedule that was inadequate to sustain performance of that response. However, the present studies were not designed to evaluate this possibility. The potential difficulty in using response rate to index associative strength has already been noted in discussions of the partial reinforcement extinction effect. However, a choice test between two responses that had identical contingentreinforcement histories but different noncontingent treatments could be used to explore this account (see Colwill, 1994).

At least two other explanations are worth considering for the findings of Experiments 1 and 2 that the strength of an $\mathrm{R}-\mathrm{O}$ association was not reduced by subsequent noncontingent presentations of $\mathrm{O}$. One possibility is that the processing of the response changes over the course of training. The idea that less attention is given to well-learned events has figured prominently in the Pearce-Hall model of Pavlovian conditioning (Pearce \& Hall, 1980). Thus, the impact of a noncontingent outcome during the initial period of acquiring an $\mathrm{R}-\mathrm{O}$ association may be more deleterious than during the later stages of training. An alternative possibility can be derived from the associative accumulation model (D. G. Davis, Staddon, Machado, \& Palmer, 1993). To the degree that subjects learn by accumulating $\mathrm{R}-\mathrm{O}$ experiences, a noncontingent relationship is going to be more difficult to detect when there is a prior history of an $\mathrm{R}-\mathrm{O}$ contingency than when there is none. Both the attentional and detection-based accounts predict, albeit for different reasons, that accompanying the introduction of the noncontingent schedule with a novel response-contingent outcome should produce some adjustment in the strength of the previously established $\mathrm{R}-\mathrm{O}$ association.

The transfer results of Experiments 1 and 3 are also pertinent to the issue of how instrumental outcomes are associated with their responses. In contrast to the $\mathrm{R}-\mathrm{O}$ account, some investigators have proposed that the outcome becomes associated with the discriminative stimulus. The expectancy generated by this Pavlovian association then comes to be associated with the instrumental response (Trapold \& Overmier, 1972). Attempts to assess the relative contribution of $\mathrm{R}-\mathrm{O}$ and $\mathrm{O}-\mathrm{R}$ associations to instrumental performance have yielded negligible support for O-R learning as a determinant of instrumental behavior (Colwill \& Triola, 2001; Rescorla, 1992b; Rescorla \& Colwill, 1989). The results of Experiment 3 agree with this conclusion. Despite the relatively higher rate of noncontingentoutcomes, the response was promoted only by presentation of an $\mathrm{S}+$ associated with the less frequent contingent outcome and not by an $\mathrm{S}+$ associated with the more frequent noncontingent outcome. An O-R account would have anticipated transfer in the latter case because of the development of an association between the noncontingent outcome expectancy and the response. Similarly, in Experiment 1, the addition of noncontingent outcomes should have increased control of the response by an expectancy of the noncontingent outcome. However, there was no significant difference in the extent to which responding was promoted by an $\mathrm{S}+$ associated with the noncontingent or contingent outcomes.

This is not to say that an expectancy of the noncontingent outcome may not have contributed at all to instrumental performance. For example, it is possible that an $\mathrm{O}-\mathrm{R}$ association may have been responsible for the marginal increases in performance observed in Experiments 3 and 4 during an $\mathrm{S}+$ that signaled the noncontingent outcome. Furthermore, an expectancy of the noncontingent outcome is likely to have played an important role in modulating development of an association between the response and the noncontingent outcome in Experiments 3 and 4. Colwill and Rescorla (1986) showed that the se- 
lective interference of noncontingent outcomes on performance of a response earning that outcome could be alleviated by signaling those noncontingent outcomes with a Pavlovian conditioned stimulus. They interpreted these data as illustrative of the fact that an earned outcome is an ineffective reinforcer when its occurrence is predicted by another cue. It seems plausible that, in Experiments 3 and 4 , the noncontingent outcome developed an association with the context that in turn undermined or blocked its ability to serve as a reinforcer for its response. However, because each outcome was presented noncontingently for one response and contingently for a different response, this analysis places some constraint on the identity of the contextual stimulus. At the very least, the response manipulandum would have to be a salient contextual feature.

In summary, these experiments establish that the rules governing acquisition of $\mathrm{R}-\mathrm{O}$ associations appear to follow a predictable pattern based on competitive models of the conditioning process (Rescorla \& Wagner, 1972). In contrast, by illustrating further the resilience of $\mathrm{R}-\mathrm{O}$ associations to a treatment designed to weaken them, the present experiments reveal the limits of the competition models in accounting for extinction of instrumental performance but preservation of the underlying association.

\section{REFERENCES}

Adams, C. D., \& Dickinson, A. (1981). Instrumental responding following reinforcer devaluation. Quarterly Journal of Experimental Psychology, 33B, 109-121.

AMSEL, A. (1958). The role of frustrative nonreward in noncontinuous reward situations. Psychological Bulletin, 55, 102-119.

Ay res, J. J., Benedict, J. O., \& Witcher, E. S. (1975). Systematic manipulation of individual events in a truly random control in rats. Journal of Comparative \& Physiological Psychology, 88, 97-103.

Benedict, J. O., \& Ayres, J. J. (1972). Factors affecting conditioning in the truly random control procedure in the rat. Journal of Comparative \& Physiological Psychology, 78, 323-330.

Bolles, R. C. (1972). Reinforcement, expectancy, and learning. Psychological Review, 79, 394-409.

Colwill, R. M. (1991). Negative discriminative stimuli provide information about the identity of omitted response-contingent outcomes. Animal Learning \& Behavior, 19, 326-336.

Colwill, R. M. (1994). Associative representations of instrumental contingencies. In D. L. Medin (Ed.), The psychology of learning and motivation (Vol. 31, pp. 1-72). New York: Academic Press.

Colwill, R. M., \& Rescorla, R. A. (1985). Post-conditioning devaluation of a reinforcer affects instrumental responding. Journal of Experimental Psychology: Animal Behavior Processes, 11, 120-132.

Colwill, R. M., \& Rescorla, R. A. (1986). Associative structures in instrumental learning. In G. H. Bower (Ed.), The psychology oflearning and motivation (Vol. 20, pp. 55-104). New York: Academic Press.

Colwill, R. M., \& Rescorla, R. A. (1988a). Associations between the discriminative stimulus and the reinforcer in instrumental learning. Journal of Experimental Psychology: Animal Behavior Processes, 14, 155-164.
Colwill, R. M., \& Rescorla, R. A. (1988b). The role of responsereinforcer associations increases throughout extended instrumental training. Animal Learning \& Behavior, 16, 105-111.

Colwill, R. M., \& Triola, S. M. (2001). Instrumental responding remains under the control of the consequent outcome after extended training. Manuscript submitted for publication.

Davis, D. G., Staddon, J. E., Machado, A., \& Palmer, R. G. (1993). The process of recurrent choice. Psychological Review, 100, 320-341.

Davis, E. R., \& Platt, J. R. (1983). Contiguity and contingency in the acquisition and maintenance of an operant. Learning \& Motivation, 14, 487-512.

Dickinson, A., \& Charnock, D. J. (1985). Contingency effects with maintained instrumental reinforcement. Quarterly Journal of Experimental Psychology, 37B, 397-416.

HAmmond, L. J. (1980). The effect of contingency upon the appetitive conditioning of free-operant behavior. Journal of Experimental Analysis of Behavior, 34, 297-304.

Mackintosh, N. J., \& Dickinson, A. (1979). Instrumental (Type II) conditioning. In A. Dickinson \& R. A. Boakes (Eds.), Mechanisms of learning and motivation (pp. 143-167). Hillsdale, NJ: Erlbaum.

Pearce, J. M., \& Hall, G. (1980). A model for Pavlovian learning: Variations in the effectiveness of conditioned but not of unconditioned stimuli. Psychological Review, 87, 532-552.

RESCORLA, R. A. (1989). Redundant treatments of neutral and excitatory stimuli in autoshaping. Journal of Experimental Psychology: Animal Behavior Processes, 15, 212-223.

RESCORLA, R. A. (1991). Associations of multiple outcomes with an instrumental response. Journal of Experimental Psychology: Animal Behavior Processes, 17, 465-474.

RESCORLA, R. A. (1992a). Response-independent outcome presentation can leave instrumental R-O associations intact. Animal Learning \& Behavior, 20, 104-111.

RESCORLA, R. A. (1992b). Response-outcome versus outcome-response associations in instrumental learning. Animal Learning \& Behavior, 20, 223-232.

Rescorla, R. A. (1993). Preservation of response-outcome associations through extinction. Animal Learning \& Behavior, 21, 238-245.

RESCORLA, R. A. (1995). Full preservation of a response-outcome association through training with a second outcome. Quarterly Journal of Experimental Psychology, 48B, 252-261.

Rescorla, R. A., \& Colwill, R. M. (1989). Associations with anticipated and obtained outcomes in instrumental learning. Animal Learning \& Behavior, 17, 291-303.

Rescorla, R. A., \& SkUCy, J. C. (1969). The effect of responseindependent reinforcers during extinction. Journal of Comparative \& Physiological Psychology, 67, 381-389.

Rescorla, R. A., \& WAGNeR, A. R. (1972). A theory of Pavlovian conditioning: Variations in the effectiveness of reinforcement and nonreinforcement. In A. H. Black \& W. F. Prokasy (Eds.), Classical conditioning II: Current research and theory (pp. 64-99). New York: Appleton-Century-Crofts.

Trapold, M. A., \& Overmier, J. B. (1972). The second learning process in instrumental learning. In A. A. Black \& W. F. Prokasy (Eds.), Classical conditioning II: Current research and theory (pp. 427452). New York: Appleton-Century-Crofts.

Wagner, A. R. (1966). Frustration and punishment. In R. N. Haber (Ed.), Current research in motivation (pp. 229-239). New York: Holt, Rinehart \& Winston.

(Manuscript received December 3, 1999; revision accepted for publication January 20, 2001.) 